\title{
The effect of stabilization exercise on pain-related disability, sleep disturbance, and psychological status of patients with non-specific chronic low back pain
}

\author{
Department of Physiotherapy, College of Medicine, University of Lagos, Lagos, Nigeria
}

Ashiyat Kehinde Akodu and Oluwagbemisola Marian Akindutire

\begin{abstract}
Background: Sleep disturbance and depression are becoming more recognized as important symptoms among individuals with chronic low back pain. This study evaluated the effect of stabilization exercise on pain-related disability, sleep disturbance and the psychological status of patients with non-specific chronic low back pain (NSCLBP).

Methods: A total of 26 patients $(\mathrm{M} / \mathrm{F}=17 / 9)$ with NSCLBP with a mean age of $50.0 \pm 15.5$ took part in this study. Participants were recruited from selected hospitals in Lagos state. Ethical approval was sought and obtained from the health research and ethics committee of Lagos University Teaching Hospital Idi-araba, Lagos, Nigeria. Participants performed stabilization exercise for eight weeks consecutively and were assessed for pain-related disability, anxiety, depression, and sleep disturbance using the pain-disability index, hospital anxiety and depression scale, and the insomnia severity index at baseline, the 4th week, and the 8th week, postintervention.
\end{abstract}

Results: The participants studied recorded significant reduction in pain-related disability $(P=0.001)$. There was also improvement in the sleep quality $(P=0.001)$, depression level $(P=0.001)$, and anxiety level $(P=$ 0.001), post intervention.

Conclusions: This study revealed that stabilization exercise is very useful in the management of sleep disturbance, pain-related disability, depression, and anxiety in NSCLBP patients. (Korean J Pain 2018; 31: 199-205)

Key Words: Anxiety; Depression; Disability evaluation; Exercise movement techniques; Low back pain; Psychological test; Sleep disorders.

\section{INTRODUCTION}

Low back pain (LBP) is a growing primary health care problem world-wide affecting over $50 \%$ of the population globally [1,2] and incurring a financial burden of at least $\$ 86$ billion annually [3]. This has led to substantial ex-

Received March 30, 2018. Revised June 2, 2018. Accepted June 4, 2018.

Correspondence to: Ashiyat Kehinde Akodu

Department of Physiotherapy, College of Medicine, University of Lagos, Lagos State 234, Nigeria

Fax: +2348034269053, E-mail: akoduashiyat@gmail.com, aakodu@unilag.edu.ng

() This is an open-access article distributed under the terms of the Creative Commons Attribution Non-Commercial License (http:// creativecommons.org/licenses/by-nc/4.0/), which permits unrestricted non-commercial use, distribution, and reproduction in any medium, provided the original work is properly cited.

Copyright (C) The Korean Pain Society, 2018 
penses for health care for the general public as a whole [4]. The bulk of people that experience LBP will improve with time; however, a significant number will suffer repeated episodes and some report continuous and persistent symptoms for many years [5]. Chronic low back pain (CLBP) is related to considerable physical and psychological disability, and is a key cause of applications for sick-leave in places of work, globally [6].

Sleep is necessary for normal functioning of the human body. Humans who experience sleep disorder have a shortened lifespan [7]. Sleep is crucial for the accurate performance of nearly all systems in the human body including the endocrine, neurological, and immune systems and it is also fundamental for memory and learning [8]. Sleep is also very important in the conservation of energy, brain plasticity, synaptic homeostasis, and neuronal recovery [9]. Particularly, lack of sleep impacts negatively on pain perception, level of functional ability, mood, and overall quality of life in patients with CLBP [10]. This condition has also been reported to be related to a reduction in functional ability, psychological status, and health-related quality of life [11].

Some exercises, such as spinal stabilization exercises, could be used to treat the stability of the spine to improve spinal control [12]. It was therefore anticipated that a specific program of stabilization exercises will most likely change the central motor control and automatically recruitment of deep core muscles in the back and abdomen [13]. A previous study has shown that stabilization exercises are more beneficial than conventional treatments to reduce pain and disability in chronic LBP patients [14].

Hence, this study was aimed at determining the effect of stabilization exercises on pain-related disability, sleep disturbance, and psychological status (depression and anxiety) in patients with NSCLBP.

\section{MATERIALS AND METHODS}

Twenty-six participants $(M / F=19 / 9)$ with ages range from 22-72 years were involved in this study. They were recruited from the outpatient physiotherapy clinics of Lagos University Teaching Hospital, Idi-Araba, Lagos, Nigeria, the Air Force Hospital Base, Ikeja, Lagos, and the Army Reference Hospital, Yaba, Lagos.

Included in this study were participants whose clinical assessment indicates that they will be able to undergo the training for stabilization exercises, subject with diagnosis of NSCLBP (this is LBP not attributed to a recognizable, known, specific pathology such as, infection, tumor, osteoporosis, lumbar spine fracture, radicular syndrome, or cauda equina syndrome) with and without radiation to the lower limbs [15].

Excluded from the study were subjects with deformities of the back (including conditions such as idiopathic adolescent scoliosis, congenital scoliosis, post-traumatic deformities, and other adult spinal deformities, including post-infective kyphosis) [16], red flags indicating serious pathology, and subjects with a history of systemic and inflammatory disease.

Assessment of participants was done by obtaining a detailed history, using an assessment form that was given to them, and through physical examinations and radiographic examination. Participants' information, such as age, sex, weight, height, duration of low back pain, and drug history, was taken.

After fulfilling the inclusion criteria, participants were fully included in the study after signing the informed consent that was given by the researcher. Ethical approval was obtained from the Health Research and Ethics Committee of Lagos University Teaching Hospital, IdiAraba, Lagos, with approval number ADM/DCST/HREC/ APP $/ 1500$.

Of the 35 participants recruited for this study, 2 were not eligible for the study, and were excluded. But only 26 participants completed the study (17 males and 9 females). Reasons for withdrawal by the remaining 7 participants were illness and travel.

Participants performed the stabilization exercises for 8 weeks, consecutively. Assessment of pain-related disability, depression, anxiety, and sleep disturbance were done using the Pain-disability Index, Hospital Anxiety and Depression Scale, and Insomnia Severity Index, pre-intervention, the 4th week, and the 8th week, post intervention.

\section{Protocol of stabilization exercises}

\section{1) Abdominal bracing}

Subjects, in a supine lying position, were instructed to perform a drawing-in maneuver of the abdomen, holding it for 8 seconds, for 30 repetitions. 


\section{2) Bracing with heel slides}

Subjects, in a supine lying position, were instructed to perform a drawing-in maneuver of the abdomen, with a heel slide on both feet for 4 seconds, for 20 repetitions.

\section{3) Bracing with leg lift}

Subjects were instructed to perform a drawing-in maneuver of the abdomen in a supine lying position, holding it while raising each leg for 4 seconds, for 20 repetitions.

\section{4) Bracing with bridging}

Subjects, in a supine lying position, were instructed to perform a drawing-in maneuver of the abdomen, gently raising the buttocks from the supporting surface and holding it for 8 seconds, for 30 repetitions.

\section{5) Bracing with bridging and leg lift}

Subjects, lying in a supine position, were instructed to perform a drawing-in maneuver of the abdomen while gently raising the buttocks from the supporting surface, holding this position while raising each leg for 8 seconds, for 30 repetitions.

\section{6) Bracing with standing}

Subjects were instructed while standing to perform a drawing-in maneuver of the abdomen for 8 seconds, for 30 repetitions.

\section{7) Quadruped arm lift with bracing (one upper limb flexed)}

Subjects were instructed, while in a prone kneeling position, to perform a drawing-in maneuver of the abdomen for 8 seconds, with one upper limb flexed, for 30 repetitions.

8) Quadruped leg lift with bracing (one lower limb is extended and lifted off the supporting surface)

Subjects were instructed, while in prone kneeling position, to perform the drawing-in maneuver of the abdomen with one of their lower limbs flexed for 8 seconds, for 30 repetitions.

9) Quadruped alternate arm and leg lift with bracing (contralateral upper and lower limbs are extended alternately)

Subjects were instructed, while in a prone kneeling position, to perform the drawing-in maneuver of the abdomen with contralateral upper and lower limbs extended alternately for 8 seconds, for 30 repetitions [17,18].

\section{Statistics}

Data were analyzed using the statistical package for social science (SPSS) version 21 and Microsoft Excel 2007, and summarized using descriptive statistics of frequency, charts, as well as mean and standard deviation.

Friedman's test was used to find the statistically significant difference in the outcome measure across the weeks, and the Wilcoxon signed rank test was used to find difference at baseline and the eighth week, post intervention.

The level of significance was set at $P<0.05$.

\section{RESULTS}

A total of 26 NSCLBP patients participated in this study. The participants consisted of 9 (34.6\%) females and 17 (65.4\%) males with a mean age of $50.08 \pm 15.45$, weight of $71.15 \pm 9.83 \mathrm{~kg}$, height of $1.70 \pm 0.83 \mathrm{~cm}$, and a BMI of $24.66 \pm 3.21 \mathrm{~kg} / \mathrm{m}^{2}$.

1. Outcome parameters at baseline, $4^{\text {th }}$ week, and $8^{\text {th }}$ week (post-intervention) of the subjects

Table 1 shows the mean score of all the outcome parameters at baseline, the 4 th week (mid intervention), and the end of the 8th week (post intervention). Friedman's test showed that there was a significant difference in all the outcome parameters only post intervention, including sleep disturbance $(P=0.001)$, pain-related disability $(P=$ $0.001)$, depression $(P=0.001)$, and anxiety $(P=0.001)$. 
Table 1. Outcome Parameters at Baseline, End $4^{\text {th }}$ Week and Post Intervention (End of $8^{\text {th }}$ Week) of the Participants

\begin{tabular}{lccccc}
\hline \multicolumn{1}{c}{ Outcome } & Pre-treatment & Mid-treatment & Post-treatment & F-value & $P$ value \\
\hline Variables & (Baseline) & (End of $4^{\text {th }}$ week) & (End of $8^{\text {th }}$ week) & & \\
& Mean $\pm S D$ & Mean $\pm S D$ & Mean $\pm S D$ & & $0.001^{*}$ \\
Sleep disturbance & $21.54 \pm 5.73$ & $16.04 \pm 3.90$ & $10.23 \pm 3.93$ & 49.52 & $0.001^{*}$ \\
Pain and disability & $34.73 \pm 11.41$ & $25.58 \pm 9.17$ & $18.08 \pm 7.53$ & 51.52 & $0.001^{*}$ \\
Depression & $8.81 \pm 2.91$ & $7.50 \pm 1.84$ & $4.65 \pm 2.21$ & 44.36 & $0.001^{*}$ \\
Anxiety & $9.54 \pm 3.17$ & $7.73 \pm 2.00$ & $5.23 \pm 2.61$ & 40.46 & \\
\hline
\end{tabular}

*Significance at $P \leq 0.05$, SD: Standard deviation, FM value: Friedman test.

Table 2. Outcome Parameters at Pre-treatment (Baseline) and Post-treatment (End of $8^{\text {th }}$ Week) of the Subjects

\begin{tabular}{lcccc}
\hline \multicolumn{1}{c}{ Outcome } & Pre-treatment & Post-treatment & Z-value & $P$ value \\
\hline Variables & (Baseline) & (End of $8^{\text {th }}$ week) & & \\
& Mean $\pm \mathrm{SD}$ & Mean \pm SD & 4.38 & $0.001^{*}$ \\
Sleep disturbance & $21.54 \pm 5.73$ & $10.23 \pm 3.93$ & 4.46 & $0.001^{*}$ \\
Pain and disability & $34.73 \pm 11.41$ & $18.08 \pm 7.53$ & 4.47 & $0.001^{*}$ \\
Depression & $8.81 \pm 2.91$ & $4.65 \pm 2.21$ & 4.22 & $0.001^{*}$ \\
Anxiety & $9.54 \pm 3.17$ & $5.23 \pm 2.61$ &
\end{tabular}

${ }^{*}$ Significance at $P \leq 0.05, \mathrm{SD}$ : Standard deviation, Z-value: Wilcoxon sign rank test.

Table 3. Correlation between Pain-disability, Sleep Disturbance and Psychological Status of the Subjects

\begin{tabular}{ccccc}
\hline \multicolumn{1}{c}{ Pain and Disability } & Correlation & $\begin{array}{c}\text { Sleep Disturbance } \\
\text { Post-Rx }\end{array}$ & $\begin{array}{c}\text { Depression } \\
\text { Post-Rx }\end{array}$ & $\begin{array}{c}\text { Anxiety } \\
\text { Post-Rx }\end{array}$ \\
\hline Post treatment (End of $8^{\text {th }}$ week) & $r$ & 0.21 & 0.42 & 0.45 \\
& $P$ value & 0.30 & $0.03^{*}$ & $0.02^{*}$
\end{tabular}

*Significance at $P \leq 0.05, \mathrm{r}$ : Pearson's correlation coefficient, Rx: Treatment.

2. Outcome parameters of the subjects at baseline and the $8^{\text {th }}$ week (post-intervention)

Table 2 shows the comparison of the mean score of all the outcome parameters at baseline and the end of the 8th week (post-intervention). Wilcoxon's sign rank test showed that there was a significant difference in all the outcome parameters assessed post intervention, including sleep disturbance $(P=0.001)$, pain-related disability $(P=$ $0.001)$, depression $(P=0.001)$, and anxiety $(P=0.001)$.

3. Correlation between the pain-related disability index, sleep disturbance, and psychological status of the subjects

Table 3 shows that there was a significant correlation between pain-related disability and depression $(P=0.03)$, as well as anxiety $(P=0.02)$, at the end of the 8th week (post-intervention).

\section{DISCUSSION}

The results of this research showed that the use of stabilization exercises is effective in the reduction of pain-related disability, sleep disturbance, and psychological status in NSCLBP patients.

The improvement in the outcome measure assessed could be due to the reestablishment of the normal control of the local muscles lumbar multifidus and transversus abdominis, which reduced the activity of more superficial muscles (rectus abdominis, external oblique, and internal oblique) which, when recruited, stabilized the spine and increased activity in the lumbar muscles.

This resulted in a decrease in pain-related disability level, which improved sleep quality and, in turn, improved 
their psychological status. Furthermore, the $\mathrm{co}^{-} \mathrm{con}-$ traction of the local muscles, such as transversus abdominis and lumbar multifidus, had been reported to be effective in the stabilization of the motion segments of the lumbar spine, particularly within the neural zone, thus providing a stable base on which the global muscles (superficial muscles) can safely act [19].

The findings of this study shows that stabilization exercises are very effective in the reduction of pain-related disability in short term intervention, which is contrary to the result of the study by Koumantakis et al. [20], who reported a reduction in the level of disability and pain in short term by their participants after undergoing general exercise than stabilization enhanced exercise. The results of this study conforms to the study of Akodu et al. [14], who reported that stabilization exercise was effective in the management of pain and functional disability in $\mathrm{pa}^{-}$ tients with NSCLBP. The findings of this study support the report of the study of Shakeri et al. [21], which focused on the effect of lumbar stabilization exercises on pain and disability in women with menstrual low back pain, and the results showed that lumbar stabilization exercises improve pain and disability. The result of this study also support the report of the study by Nava-Bringas et al. [22], who, in their study on the adherence to a stability exercise program in patients with CLBP, reported that there was reduction in pain, with functional improvement, and that the improvement presented more quickly than the control following adherence to a lumbar stabilization exercise program.

The result of this study revealed that there was a statistically significant improvement in the psychological status (depression and anxiety) of the subjects; this could be as a result of reduction in the pain-related disability of the participants after undergoing a regimen of stabilization exercises. This finding supports the assertion of Balasubramaniam et al. [23], who reported that depression reduces once there is a change in the perception of pain and disability.

The study of Shakeri et al. [21], presented positive outcomes in the stabilization exercise groups due to activation of the deep stabilizer muscles during the motor control exercises. Antunes et al. [24], conducted a cross-sectional study on the relationship between chronic low back pain and depression, as well as kinesiophobia. It was concluded that the prevalence of depression is high in patients with
CLBP, and that their depression is associated with poor quality of life. However, in the study of Akodu et al. [18], it was reported that stabilization exercise is effective in the improvement of the psychological aspect of quality of life, as well as the social status of patients with NSCLBP. This result also agrees with the report of the study of Sethi [25], who studied the impact of short duration (4 weeks) core stability exercises on depression, anxiety and stress status in adult patients with CLBP. They concluded that there was a significant improvement after 4 weeks of core stability training in the depression, anxiety, and stress level of the participants.

A previous study has indicated that low back pain was associated with patients' emotional status [26]. Depression also has a bidirectional relationship with pain [27]. Bair et al. [28], demonstrated that depression is associated with more pain sites, greater pain intensity, longer duration of pain, and an increased risk of poor treatment response. Depression produces substantial disability and a decrease in health-related quality of life [29]. The study of Hung et al. [30], found that depression was the most powerful factor associated with disability in LBP patients; more so, insufficient sleep may result in depression [31].

According to the result of this study, there was a statistically significant improvement in the sleep disturbance of patient with NSCLBP; this could be as a result of reduction in pain and disability which in turn improves their sleep quality. Pain is associated with sleep; pain disturbs sleep, while sleep disturbance also increases pain intensity and decreases the ability to cope with pain [32]. The occurrence of sleep disturbance increases tiredness, sleeping during the day and low mood, which may lead to harmful impact such as weakening memory, barrier to work, stress in daily life, poor quality of life, and more severe pain in individuals [33]. Disturbance in sleep patterns is commonly seen in people with anxiety and related disorders; research has shown that sleep disturbance may predict the development of anxiety disorders [34]. The development of sleep disturbance is preceded by anxiety and depression in young women, whereas anxiety and sleep had a bidirectional relationship in men and older women [35], which concurs with the result of this study on the age-gender population of older men and women. A study by Lee et al. [36], also reported that patients who had subjective sleep disturbance were more likely to develop moderate to severe depression. 
Therefore, stabilization exercise can cause a reduction in pain which would, in turn, reduce disability caused by pain, sleep disturbance, depression, and anxiety, since they are all multidirectional. Rapt attention should be given to all these variables (sleep disturbance, depression, anxiety, and pain-related disability) during management, and outcome measures should be incorporated in the assessment of them in patients.

The outcome of this study showed a statistically significant correlation between pain-related disability, depression, and anxiety level post intervention (at the end of 8th week) which could be as a result of re-establishment of the normal control of the local muscles of the back. But it showed no significant relationship between pain, disability, and sleep disturbance post treatment. This might be because a majority of the participants fell into the category of sub-threshold insomnia even after undergoing 8 weeks of stabilization exercises. This agrees with the results of the study of Wang et al. [37], which stated that the pain intensity of LBP was correlated with the level of depression and anxiety. But it disagrees with their findings which state that the sleep disturbance score was significantly correlated with the pain intensity of LBP patients. This result also supports Kelly et al. [38], who revealed in their study that sleep disturbance, depression, anxiety, and pain intensity were correlated with each other.

For instance, sleep disturbance might aggravate symptoms of pain; conversely, symptoms of pain might trigger sleep disturbance [39]. The study of O'Donoghue et al. [40], also suggested that sleep disturbance is slightly associated with pain intensity compared to its strong association with psychological status and poor mood. The study was limited in terms of long term follow up to determine if there would be consistency in the results and also improvement in the study population.

In conclusion, the results of this study provide evidence that patients with NSCLBP can achieve significant benefit from stabilization exercise in regards to the level of their pain-related disability, sleep disturbance, and psychological status (depression, anxiety). Therefore, attention should be given to all these variables (sleep disturbance, depression, anxiety, and pain-related disability) during management, and outcome measures should be incorporated into the assessment of these patients.

\section{ACKNOWLEDGEMENTS}

The authors appreciate and acknowledge the patients that participated in this study.

\section{REFERENCES}

1. Freburger JK, Holmes GM, Agans RP, Jackman AM, Darter JD, Wallace AS, et al. The rising prevalence of chronic low back pain. Arch Intern Med 2009; 169: 251-8.

2. Hanney WJ, Kolber MJ, Beekhuizen KS. Implications for physical activity in the population with low back pain. Am J Lifestyle Med 2009; 3: 63-70.

3. Gore M, Sadosky A, Stacey BR, Tai KS, Leslie D. The burden of chronic low back pain: clinical comorbidities, treatment patterns, and health care costs in usual care settings. Spine (Phila Pa 1976) 2012; 37: E668-77.

4. Manchikanti L, Singh V, Datta S, Cohen SP, Hirsch JA; American Society of Interventional Pain Physicians. Comprehensive review of epidemiology, scope, and impact of spinal pain. Pain Physician 2009; 12: E35-70.

5. Dunn KM, Croft PR. Epidemiology and natural history of low back pain. Eura Medicophys 2004; 40: 9-13.

6. Dunn KM, Jordan KP, Croft PR. Contributions of prognostic factors for poor outcome in primary care low back pain patients. Eur J Pain 2011; 15: 313-9.

7. Kundermann B, Krieg JC, Schreiber W, Lautenbacher S. The effect of sleep deprivation on pain. Pain Res Manag 2004; 9: 25-32.

8. Brand S, Kirov R. Sleep and its importance in adolescence and in common adolescent somatic and psychiatric conditions. Int J Gen Med 2011; 4: 425-42.

9. Naughton F, Ashworth P. Skevington SM. Does sleep quality predict pain-related disability in chronic pain patients? The mediating roles of depression and pain severity. Pain 2007; 127: 243-52.

10. van de Water AT, Eadie J, Hurley DA. Investigation of sleep disturbance in chronic low back pain: an age- and gender-matched case-control study over a 7-night period. Man Ther 2011; 16: 550-6.

11. de Paula Lima PO, de Oliveira RR, Costa LO, Laurentino GE. Measurement properties of the pressure biofeedback unit in the evaluation of transversus abdominis muscle activity: a systematic review. Physiotherapy 2011; 97: 100-6.

12. Millisdotter M, Strömqvist B. Early neuromuscular customized training after surgery for lumbar disc herniation: a prospective controlled study. Eur Spine J 2007; 16: 19-26.

13. Akodu AK, Akinbo SR, Odebiyi DO. Effect of stabilization exercise on pain and disability in patients with chronic low back pain. Indian J Physiother Occup Ther 2015; 9: 170-5.

14. Akodu AK, Akinbo SR, Odebiyi DO. Effect of stabilization 
exercise on lumbar multifidus muscle thickness in patients with non-specific chronic low back pain. Iran Rehabil J 2014; 12: 6-10.

15. Balagué F, Mannion AF, Pellisé F, Cedraschi C. Clinical update: low back pain. Lancet 2007; 369: 726-8.

16. Moon MS, Lee BJ, Kim SS. Spinal deformity. Indian J Orthop 2010; 44: 123-6.

17. Hicks GE, Fritz JM, Delitto A, McGill SM. Preliminary development of a clinical prediction rule for determining which patients with low back pain will respond to a stabilization exercise program. Arch Phys Med Rehabil 2005; 86: 1753-62.

18. Akodu AK, Tella BA, Olujobi OD. Effect of stabilization exercise on pain and quality of life of patients with non-specific chronic low back pain. Afr J Physiother Rehabil Sci 2015; 7: 7-11.

19. Wilke HJ, Wolf S, Claes LE, Arand M, Wiesend A. Stability increase of the lumbar spine with different muscle groups. A biomechanical in vitro study. Spine (Phila Pa 1976) 1995; 20: 192-8.

20. Koumantakis GA, Watson PJ, Oldham JA. Trunk muscle stabilization training plus general exercise versus general exercise only: randomized controlled trial of patients with recurrent low back pain. Phys Ther 2005; 85: 209-25.

21. Shakeri $H$, Fathollahi Z, Karimi N, Arab AM. Effect of functional lumbar stabilization exercises on pain, disability, and kinesiophobia in women with menstrual low back pain: a preliminary trial. J Chiropr Med 2013; 12: 160-7.

22. Nava-Bringas TI, Roeniger-Desatnik A, Arellano-Hernández A, Cruz-Medina E. Adherence to a stability exercise program in patients with chronic low back pain. Cir Cir 2016; 84: 384-91.

23. Balasubramaniam A, Bharathi M. Effect of motor control exercises on psychological variables in chronic low back pain in computer professionals. Int J Pharma Bio Sci 2016; 7: 490-4.

24. Antunes RS, de Macedo BG, Amaral TS, Gomes HA, Pereira LS, Rocha FL. Pain, kinesiophobia and quality of life in chronic low back pain and depression. Acta Ortop Bras 2013; 21: 27-9.

25. Sethi $V$, Pragyadeep. Impact of short duration (4 weeks) core stability exercise on depression, anxiety and stress status of adult patients with chronic low back pain. J Pharm Biomed Sci 2012; 23: 1-4.

26. Ramond A, Bouton C, Richard I, Roquelaure Y, Baufreton C, Legrand $E$, et al. Psychosocial risk factors for chronic low back pain in primary care--a systematic review. Fam Pract 2011; 28: 12-21.

27. Sribastav SS, Peiheng H, Jun L, Zemin L, Fuxin W, Jianru W, et al. Interplay among pain intensity, sleep disturbance and emotion in patients with non-specific low back pain.
PeerJ 2017; 5: e3282.

28. Bair MJ, Robinson RL, Katon W, Kroenke K. Depression and pain comorbidity: a literature review. Arch Intern Med 2003; 163: $2433-45$.

29. Bair MJ, Wu J, Damush TM, Sutherland JM, Kroenke K, Association of depression and anxiety alone and in combination with chronic musculoskeletal pain in primary care patients. Psychosom Med 2008; 70: 890-7.

30. Hung Cl, Liu CY, Fu TS. Depression: an important factor associated with disability among patients with chronic low back pain. Int J Psychiatry Med 2015; 49: 187-98.

31. Xu Z, Su H, Zou Y, Chen J, Wu J, Chang W. Sleep quality of Chinese adolescents: distribution and its associated factors. J Paediatr Child Health 2012; 48: 138-45.

32. Schuh-Hofer S, Wodarski R, Pfau DB, Caspani O, Magerl W, Kennedy JD, et al. One night of total sleep deprivation promotes a state of generalized hyperalgesia: a surrogate pain model to study the relationship of insomnia and pain. Pain 2013; 154: 1613-21.

33. Sezgin M, Hasanefendioğlu EZ, Sungur MA, Incel NA, Çimen ÖB, Kan i k A, et al. Sleep quality in patients with chronic low back pain: a cross-sectional study assesing its relations with pain, functional status and quality of life. J Back Musculoskeletal Rehabil 2015; 28: 433-41.

34. Batterham PJ, Glozier N, Christensen H. Sleep disturbance, personality and the onset of depression and anxiety: prospective cohort study. Aust N Z J Psychiatry 2012; 46: 1089-98.

35. Spira AP, Stone K, Beaudreau SA, Ancoli-Israel S, Yaffe K. Anxiety symptoms and objectively measured sleep quality in older women. Am J Geriatr Psychiatry 2009; 17: 136-43.

36. Lee HJ, Choi EJ, Nahm FS, Yoon IY, Lee PB. Prevalence of unrecognized depression in patients with chronic pain without a history of psychiatric diseases. Korean $J$ Pain 2018; 31: 116-24.

37. Wang HY, Fu TS, Hsu SC, Hung Cl. Association of depression with sleep quality might be greater than that of pain intensity among outpatients with chronic low back pain. Neuropsychiatr Dis Treat 2016; 12: 1993-8.

38. Kelly GA, Blake C, Power CK, O'keeffe D, Fullen BM. The association between chronic low back pain and sleep: a systematic review. Clin J Pain 2011; 27: 169-81.

39. Siengsukon C, Emmanuel NM, Sharma NK. Relationship between low back pain and sleep quality. J Nov Physiother 2013; 3: 168.

40. O'Donoghue GM, Fox N, Heneghan C, Hurley DA. Objective and subjective assessment of sleep in chronic low back pain patients compared with healthy age and gender matched controls: a pilot study. BMC Musculoskelet Disord 2009; 10: 122. 